\title{
Model-based Automatic Segmentation of Ascending Aorta from Multimodality Medical Data
}

\author{
Noha A. Seada, Safwat Hamad, Mostafa G. M. Mostafa \\ Sciencitifc Computing and Computer Science Departments, Faculty of Computer and Information Sciences, \\ Ain Shams University, Egypt
}

\begin{tabular}{l}
\hline Article Info \\
\hline Article history: \\
Received Sep 12, 2016 \\
Revised Nov 13, 2016 \\
Accepted Nov 27, 2016 \\
\hline
\end{tabular}

Keyword:

Angiography (CTA)

Ascending aorta segmentation

Automatic segmentation

Computed tomography

Phase-contrast magnetic

Resonance images (PC-MRI)

Seeded region growing

\begin{abstract}
Automatic Ascending Aorta Segmentation is one of the important steps towards automatic segmentation of the whole cardiac tree. This paper presents a novel approach for the automatic segmentation of the ascending aorta from two imaging modalities: CTA (Computed Tomography Angiography) and PC-MRI (Phase-Contrast Magnetic Resonance Images). The novel approach is an algorithm that works without the need for setting manual seed points or applying preprocessing steps or setting a region of interest. Instead, the proposed algorithm automatically detects and segments the ascending aorta using an ascending aorta model built from its anatomical features. The proposed segmentation algorithm begins with aorta detection through features model fitting augmented with Hough transform, where the ascending aorta is identified from the descending aorta and any other circular structures based on the proposed model. After detection, the whole ascending aorta is segmented up from the aortic arch down to the ostia points using a novel automatic seeded region growing algorithm. The proposed algorithm is fully automatic, works in real-time and robust as parameters used are the same for all the tested datasets. The detection and segmentation of the ascending aorta succeeded in all test cases acquired from the two imaging modalities; proving the robustness of the proposed ascending aorta model and algorithm for the automatic segmentation process even on data from different modalities and different scanner types. The accuracy of the segmentation has a mean Dice Similarity Coefficient (DSC) of $94.72 \%$ for CTA datasets and $97.13 \%$ for PC-MRI datasets.
\end{abstract}

Copyright $\odot 2016$ Institute of Advanced Engineering and Science. All rights reserved.

\section{Corresponding Author:}

Noha A. Seada,

Sciencitifc Computing and Computer Science Departments,

Faculty of Computer and Information Sciences,

Ain Shams University, Egypt.

Email: noha_sabour@cis.asu.edu.eg

\section{INTRODUCTION}

For cardiologists; inspecting the vascular structures is of very high importance to assess patient's cardiovascular risk. Current developmental techniques in imaging modalities including CTA (Computed Tomography Angiography) and PC-MRI (Phase-Contrast Magnetic Resonance Images) has brought attention to the possibility for non-invasive assessment of CAD (Coronary Artery Disease) patients. This paper focuses on segmenting the beginning of the cardiac tree which is the ascending aorta beginning from the aortic arch down to the ostia points (from which the coronaries emerge), see Figure 1; from both CTA and PC-MRI images. Our main objective is to achieve accurate segmentation of the ascending aorta to help in multiple further processing. In CTA images being able to automatically segment the ascending aorta and determine the ostia locations that reside at the lateral sides of the segmented ascending aorta; will lead to efficient automatic seeding for heart coronaries segmentation. And for PC-MRI images; the accurate segmentation of the 
ascending aorta helps quantify blood flow for assessing the cardiovascular system looking for some diseases like aortic narrowing known as Aortic Coarctation [1]. For the automatic segmentation process; ascending aorta features model is proposed, that is built based on the ascending aorta anatomical features. These features are shape (circular/elliptical), size, anatomical location and intensity. The segmentation algorithm goes through two main stages: Partial and Full ascending aorta segmentation. In the first stage; the ascending aorta is detected and identified from other similar structures using Hough transform for detecting circular/elliptical structures augmented with the model of the ascending aorta. The output of the first stage is part of the ascending aorta being segmented (this part of the ascending aorta where its shape is uniformly circular). In the second stage, the remaining part of the ascending aorta is segmented using a proposed modified version of the seeded region growing algorithm; center to edge ray-search-based propagation region growing. The segmentation on both imaging modalities has been validated versus manual segmentation and dice similarity coefficient is calculated. The proposed segmentation algorithm succeeded to detect and segment the ascending aorta, with same parameters value set over all the test cases, even on datasets with artifacts (as indicated in the results and discussion section). This proves the robustness of the algorithm, in addition to the accuracy attained proves the reliability of the approach for clinical use.

Segmentation of the ascending aorta methodologies mentioned in literature varies from semiautomatic to automatic methodologies. Semi-automatic methodologies mainly rely on a manual seed point entered by the user in the middle of the aorta or on the aorta top slice. Some other methodologies define a region of interest of vascular structures in order to minimize the searching area for the aorta segmentation process. From the semi-automatic methodologies mentioned in literature: Xie et al. [2] begin their segmentation by setting a manual seed point inside the aorta lumen, then used a cylindrical model fitting in the 3D image space to track the aorta centerline. Bock et al. [3] used a region growing algorithm for segmenting the aorta and obtained its seed by manually selecting a single seed point inside the ascending aorta above the coronary ostia locations, while Hennemuth et al. [4] manually select the seed point in the middle of the aorta above the first coronary artery. Both Kitslaar et al. [5] and Bouraoui et al. [6] isolated the heart region as their region of interest; so as to minimize the computational cost. Kitslaar et al. used the Hough transform algorithm to detect the ascending aorta as circles and they isolated the heart region using a2D balloon model inflation. They then used gradient information to obtain heart contour and applied connected component analysis on the isolated heart region and created a list of vessels candidate list. They used the top two points from that list as the stopping criteria for the region grower that segments the ascending aorta. Bouraoui et al. down sampled the input images for cost reduction and applied Gray-level hit-or-miss transform (GL HMT) in conjunction with different structuring elements for segmenting the heart region, the aorta and the coronaries.

From the automatic segmentation methodologies is the one presented by Saur et al. [7]. They began their search in $\mathrm{x}$ and $\mathrm{y}$ directions in every CTA image slice and used a two-level threshold to find the aorta circle circumference. Although their work succeeded for detecting the ascending aorta circumference, their work would have been more efficient; if they have relied on the anatomical features of the ascending aorta (like ascending aorta geometric shape, diameter range and location with respect to other organs) that would have helped them minimize iterations till finding the ascending aorta circumference. All the previous work mentioned is applied on CTA datasets, while Herment et al. [8] and Al-Agamy at al. [9] segmented the aorta from PC-MRI images. Herment et al. begins the segmentation process by manually defining a rectangular region of interest containing the aorta. The dynamic time frames of the PC-MRI images are averaged overtime within that region. They then use a $2 \mathrm{D}+\mathrm{t}$ deformable surface model to segment the aorta. Al-Agamy at al. implements semi-automated segmentation by asking the user to click two points inside the aorta. Afterwards they segment the ascending and descending aorta cross sections using a modified version of the active contour models.

After analyzing the previous work in the area of ascending aorta segmentation, it was obserevd that using manual seed points yields a solution that yet needs user interaction and the results of the algorithm is highly dependent on an accuarate initialization. Also, segmentation methodologies that needs a region of interest (ROI) to be specified at the beginning; adds extra processing to the segmentation pipeline and also the results will depend on the accurate ROI specification. Moreover, automatic methodologies that search the whole image for detecting the aorta region goes into multiple iterations till finding the aorta circumference which results into long processing times. This in addition to using multiple thresholds that need value-tuning for images of different volumes and acquired using different scanner types; yeilds a non-generic segmentation algorithm.

In this paper, a fully automatic methodology is presented that avoids all the drawbacks observed in the previous work. The ascending aorta is segmented without the need of any manual seed points, or a region of interest to be defined, or blind scanning of the image slice in all directions to search for the aorta region. We rather defined an ascending aorta features model that is augmented with our proposed methodolgy and yields successful automatic detection and identification of the ascending aorta in an efficient way in all the datasets

IJECE Vol. 6, No. 6, December 2016 : $3161-3173$ 
provided from the two imaging modalities (CTA/PC-MRI) and this is proved by the experimental results and comparison done with previous work in terms of accuracy and processing times.

The paper is organized as follows: Section 2 presents the proposed automatic ascending aorta segmentation methodology. Section 3 presents the results and discussion and performance measures (accuracy and processing time). Finally conclusion is presented in Section 4.

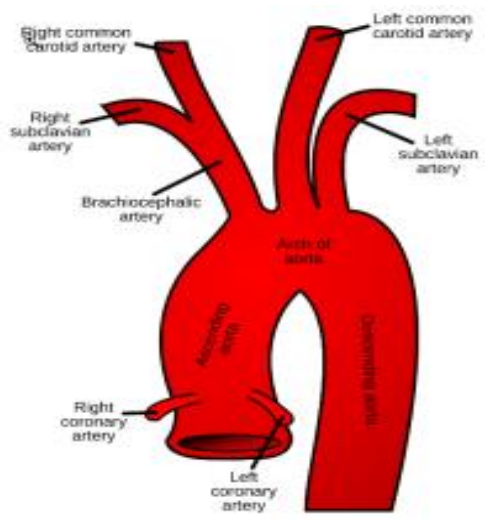

Figure 1. The ascending aorta depicted from the aortic arch down to ostia points [10]

\section{PROPOSED ASCENDING AORTA AUTOMATIC SEGMENTATION APPROACH}

In this section, the proposed approach for the automatic segmentation of the ascending aorta is presented. The ascending aorta is circular/elliptical in shape in multiple sequential images (beginning from the cranial third of the volume) [7], [11], and then its shape begins to deform in later consequent images. Based on this observation the proposed method for automatically segmenting the full ascending aorta is divided into two main stages; partial and full segmentation, see Figure 2. The output of the first stage is part of the uniformly circular ascending aorta being segmented. In the second stage, the segmented part is used as a seed for segmenting the remaining ascending aorta part to achieve full segmentation of the ascending aorta from the aortic arch down to the ostia points. Moreover to minimize computations complexity and to successfully identify the ascending aorta from other similar structures in the image, an ascending aorta model is proposed. The model helps in localizing and identifying the ascending aorta in a quick manner with no need to set a region of interest or do any preprocessing on the volume before the ascending aorta segmentation and performance is yet real-time.

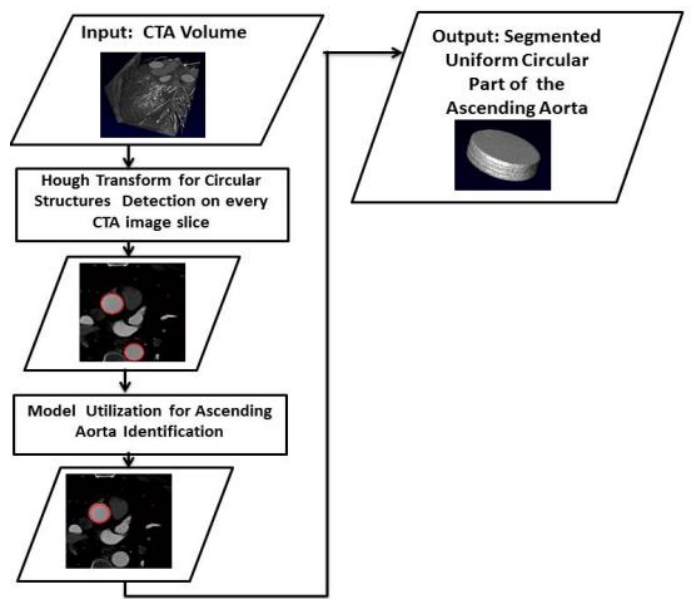

(a) Partial Ascending Aorta Segmentation

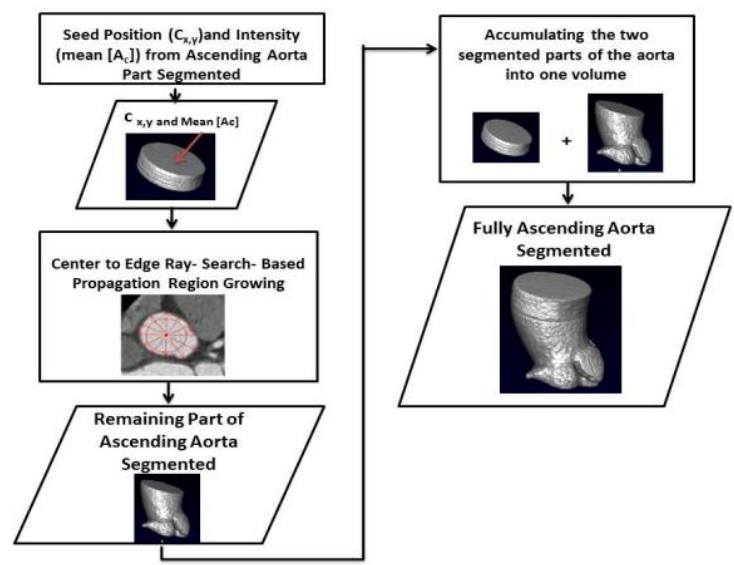

(b) Full Ascending Aorta Segmentation

Figure 2. Ascending Aorta Segmentation Flow Chart for Partial and Full Segmentation Stages 


\subsection{Proposed Ascending Aorta Model}

To correctly detect the ascending aorta cross section from any other organs that are similar in structure makes building an ascending aorta model an essential addition to the detection and segmentation processes. The proposed model of the ascending aorta is built based on its anatomical features. These anatomical features are: geometric shape, size, anatomical location with respect to other organs and intensity values.

1. Shape: The ascending aorta has circular/elliptical shape [7], [12] from the aortic arch down to ostia points, see Figure 1.

2. Size: The ascending aorta diameter is bigger than that of the descending aorta in healthy patients. The normal diameter of the ascending aorta is less than $2.1 \mathrm{~cm} / \mathrm{m}^{2}$ and the normal diameter of the descending aorta is less than $1.6 \mathrm{~cm} / \mathrm{m}^{2}$ [13].

3. Anatomical Location: The ascending aorta anatomical location with respect to other organs; is the nearest to the center of gravity of the image. For every detected circular structure in the image, the ascending aorta will be the circular structure with the minimum Euclidean distance to the image center.

$$
\text { Euclidean distance }=\sqrt{\left(C_{I x}-C_{c x}\right)^{2}+\left(C_{I y}-C_{c y}\right)^{2}}
$$

where $\mathrm{C}_{\mathrm{Ix}}, \mathrm{C}_{\mathrm{Iy}}$ and $\mathrm{C}_{\mathrm{cx}}, \mathrm{C}_{\mathrm{cy}}$ are the center points of image (I) and detected circles (c) on that image slice.

4. Intensity: In CTA images the vessels are injected with a contrast agent, thus the ascending aorta intensity has a range of Hounsfield values [14] that is used as a threshold in the segmentation algorithm and in the PC-MRI images an intensity range is empirically determined (after training the datasets) as a threshold for the segmentation as well.

\subsection{Partial Ascending Aorta Segmentation}

From the ascending aorta flow chart depicted in Figure 2, the first stage of the automatic segmentation process is the partial ascending aorta segmentation. It begins with circular structures detection in each CTA/PC-MRI image slice, and then the ascending aorta is identified from any other circular structure using the ascending aorta model. The detection and identification begin from the cranial third of the CTA volume [11]. In the beginning of this part of the volume, the ascending aorta appears as a uniform circle and accordingly could be segmented with any algorithm for detecting circles like the famous 2D Hough Transform, see Figures 3-a and 3-c. The segmentation will proceed to segment the ascending aorta from consecutive CTA/PC-MRI image slices and will stop as soon as the ascending aorta shape is no more a uniform circle. Finally, the output of this stage is a part of the ascending aorta being automatically segmented.

\subsubsection{Hough Transform for Circular Structures Detection}

The Hough transform is mainly used for detecting circular structures in the image [11], [15-16]. It detects circles with known radius range and thus it fits to our problem [17]. The Hough transform is applied on CTA/MRI image slices beginning from the cranial third of the CTA volume. The Hough transform fits in this part of the segmentation pipeline; as the ascending aorta is uniformly circular in that part of the volume, see Figure 3(a, c, d).

\subsection{Model Utilization for Ascending Aorta Identification}

After applying Hough transform; all circular structures in the image are detected. These circular structures would be the ascending aorta, descending aorta or even the spinal cord cross section. Therefore to identify the ascending aorta among other circular structures, model fitting is done on the output of the Hough transform. After applying this combination between the Hough transform and the ascending aorta model on consecutive CTA/PC-MRI image slices; the ascending aorta cross section is successfully identified and finally a part of the ascending aorta is segmented in a computationally efficient manner, see Figure 3(d). 


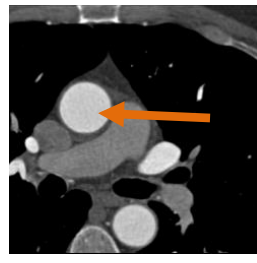

(a)

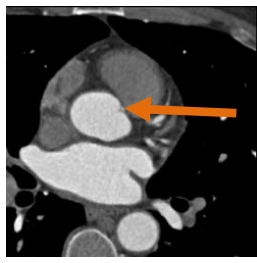

(b)

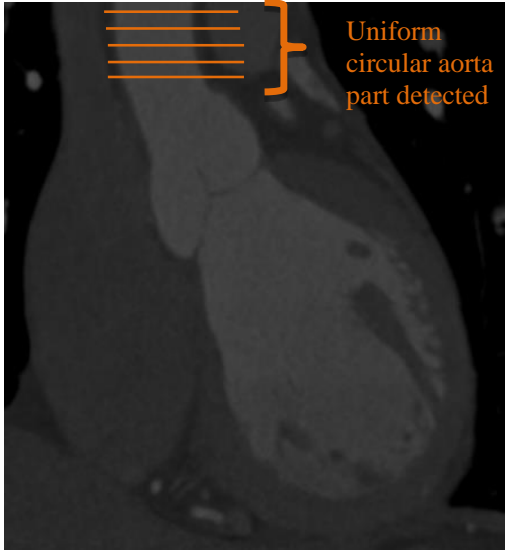

(c)

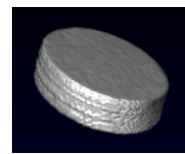

(d)

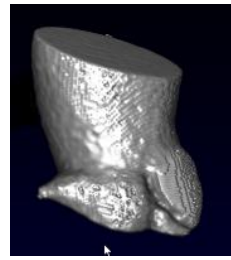

(e)

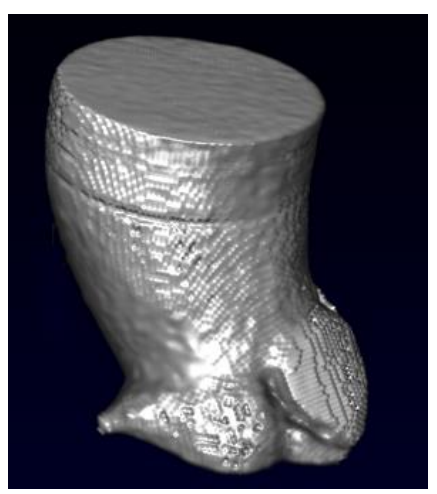

(f)

Figure 3. (a) Ascending Aorta Appears as a Uniform Circle, (b) Ascending Aorta Shape Appears Non-Uniform, (c) Sagittal View for the Heart Showing the Full Ascending Aorta Shape, (d) The Uniform Circular Part of the Ascending Aorta Segmented in First Stage, (e) The Remaining (Non-Uniform) Part of the Ascending Aorta Segmented in Stage 2, (f) Full Ascending Aorta Segmented

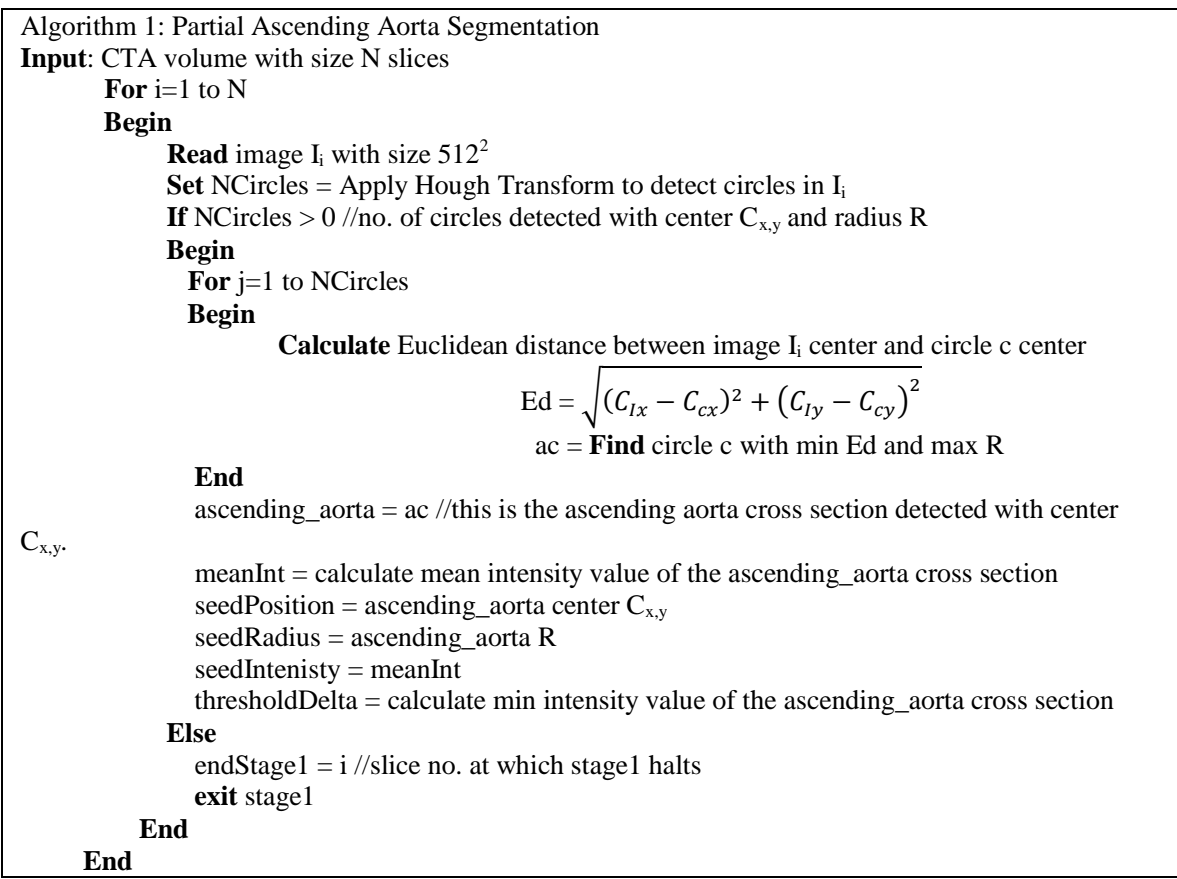

Figure 4. Pseudo Code for Partial Ascending Aorta Segmentation Stage

\subsection{Full Ascending Aorta Segmentation}

The automatic segmentation of the remaining part of the ascending aorta begins by taking the output of the first stage as a seed for the segmentation process. When the ascending aorta shape begins to deform and is no more circular, see Figure 3(b); the first stage halts since the Hough transform is sensitive to shape deviation and noise [15] and failed to detect the ascending aorta cross sections in that case. So, the second stage begins to segment the remaining ascending aorta part up to the ostia locations. In the second stage a modified version of the seeded region growing approach is proposed. The proposed algorithm is called center to edge ray-search-based propagation region growing approach. The proposed algorithm takes the seed position and intensity of the ascending aorta from the first stage and begins searching every new unvisited 
slice for the ascending aorta cross section based on the seed position and intensity (region growing threshold). In each image slice, the search begins from the seed position (center point) and propagates as a ray checking every pixel along that ray whether it belongs to the ascending aorta or not. The ray stops propagating when it hits an edge (the ascending aorta edge). The rays move in a circular fashion to scan the whole ascending aorta cross section from $0^{\circ}$ to $360^{\circ}$, see Figure 5. The seed position and intensity are then updated to be used by the region growing algorithm in the consecutive slice. This presents a kind of learning from the volume itself to help in accurately segmenting the ascending aorta. The seed taken from the first stage helped simplify the region growing algorithm work flow and raises the computation efficiency of the segmentation process.

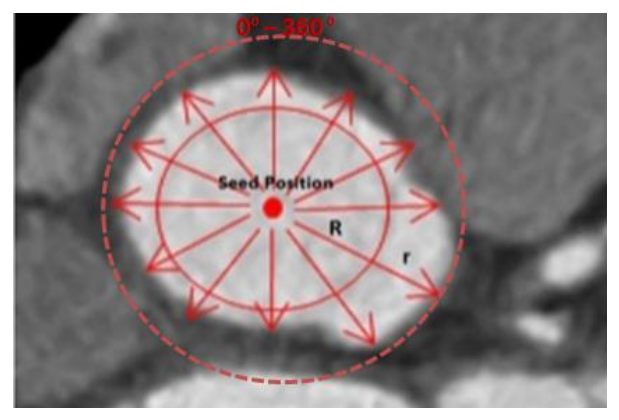

Figure 5. Region Growing Rays Propagate from Center Seed Position Till Hitting an Edge with a Max Ray Length of $(\mathrm{R}+\mathrm{r})$, moving in a Circle from Angle $0^{\circ}$ to $360^{\circ}$

\subsubsection{Center to Edge Ray-Search-Based Propagation Region Growing Algorithm}

Seeded region growing is a robust and computation efficient technique used for segmenting a region of interest from an image and the accuracy of the results are based on the right choice of the seed(s) [18]. For satisfying the intensity and connectivity criteria; the seeded region growing algorithm requires seed position, seed intensity, and expansion and termination criteria. All these inputs for the region growing algorithm are taken from the output of the first stage as follows:

1. Seed position: center $\mathrm{C}_{\mathrm{x}, \mathrm{y}}$ of the top detected ascending aorta cross section resulting from stage 1 .

2. Seed intensity: mean intensity mean $\left[\mathrm{A}_{\mathrm{c}}\right]$ of the top detected ascending aorta cross section resulting from stage1.

3. Expansion criteria on a newly given image slice: region growing rays propagate from center seed position till hitting an edge, moving in a circle from angle $0^{\circ}$ to $360^{\circ}$.

4. Termination criteria: max ray length is $(R+r)$, where $R$ is the radius of the top detected ascending aorta cross section resulting from stage 1 and $\mathrm{r}$ is a parameter beginning with value 1 and incremented while the intensity value of the visited pixel is less than a specified threshold.

2.3.2. The center to edge ray-search-based propagation region growing algorithm works as follows:

Let $\mathrm{I}$ be the image slice under inspection and $\mathrm{A}_{\mathrm{c}}$ represent the set of pixel values of the top detected ascending aorta circle with center $\mathrm{C}_{\mathrm{x}, \mathrm{y}}$ and radius $\mathrm{R}$, then:

$$
\text { Seed Intensity }=\text { mean }\left[A_{c}\right] \text {, Seed position }=C_{x, y} .
$$

1. The seed position $\left(\mathrm{C}_{\mathrm{x}, \mathrm{y}}\right)$ and seed intensity (mean $\left[\mathrm{A}_{\mathrm{c}}\right]$ ) are taken from the top detected ascending aorta cross section resulting from the partial segmentation stage.

2. For searching in a new consecutive CTA image slice, a group of rays (360 rays moving in a circular fashion with angle $\left.\theta_{\mathrm{A}}=\left(0^{\circ}-360^{\circ}\right)\right)$ propagates from the seed position till hitting an edge, see Figure 5 .

3. Since the search is in a circular fashion, every new pixel $\mathrm{P}_{\mathrm{x}, \mathrm{y}}$ position (the pixel to be tested for being an ascending aorta pixel) is found by the circle equation:

$$
\begin{aligned}
& P_{x}=C_{x}+\left((R+r) * \cos \left(\theta_{A}\right)\right) \\
& P_{y}=C_{y}+\left((R+r) * \sin \left(\theta_{A}\right)\right)
\end{aligned}
$$


4. Every pixel visited by the ray is tested for being an ascending aorta pixel (comparing it with the seed intensity provided). The new pixel $\mathrm{P}_{\mathrm{x}, \mathrm{y}}$ is added to the set $\mathrm{A}_{\mathrm{c}}$ of the new ascending aorta cross section if its intensity $I\left(P_{X}, P_{y}\right)$ satisfies the following conditions:

$$
\begin{aligned}
& I\left(P_{X}, P_{y}\right) \in\left\{A_{C}\right\} \\
& \text { if }\left\{\begin{array}{l}
I\left(P_{X}, P_{y}\right)>0, \\
\left|I\left(P_{X}, P_{y}\right)-\operatorname{mean}\left[A_{C}\right]\right|>\delta
\end{array}\right.
\end{aligned}
$$

The first condition $I\left(P_{X}, P_{y}\right)>0$ is essential for avoiding leakage outside the edge of the ascending aorta region (as the houns field unit [14] values of pixels at the outer side of the edge are negative). Also, the pixel is an aorta pixel if the difference between its intensity and that of the mean intensity $\operatorname{mean}\left[A_{c}\right]$ is greater than delta $\delta$, where $\delta$ is a thresholding parameter representing the minimum houns field value calculated from the top detected ascending aorta cross section resulting from stage 1 . These two intensity conditions secure that the new pixel added lies inside the aorta cross section.

5. If the pixel is an ascending aorta pixel, it's added to the set $\left\{A_{C}\right\}$ that will constitute the new segmented ascending aorta cross section.

6. Finally the center $\mathrm{C}_{\mathrm{x}, \mathrm{y}}$ and seed intensity (mean $\left[\mathrm{A}_{\mathrm{c}}\right]$ ) are updated for consecutive slices search.

The proposed ray propagation approach guarantees that no pixel is visited or tested more than once and this enhances the performance. Moreover it succeeds to give us significant results for the ascending aorta segmentation, see Results section. Also, the approach is promising for segmenting the ascending aorta with dissection or aneurysm; through controlling the parameter $\mathrm{r}$. Although any algorithm is challenged by the number of parameters to tune; our approach have only one parameter; $r$ to control and it's not empirical but rather controlled by the approximate radius value of the ascending aorta, and is increased in incremental steps and terminated with the conditions in Equation 4. Finally to cover all conditions, it is important to mention that if the right seed point is not provided from stage 1(in case stage 1 failed to detect any circular ascending aorta cross section); the user will be prompted to select a seed point to be used by the region growing algorithm. Figure 6 shows pseudo code for full ascending aorta segmentation stage

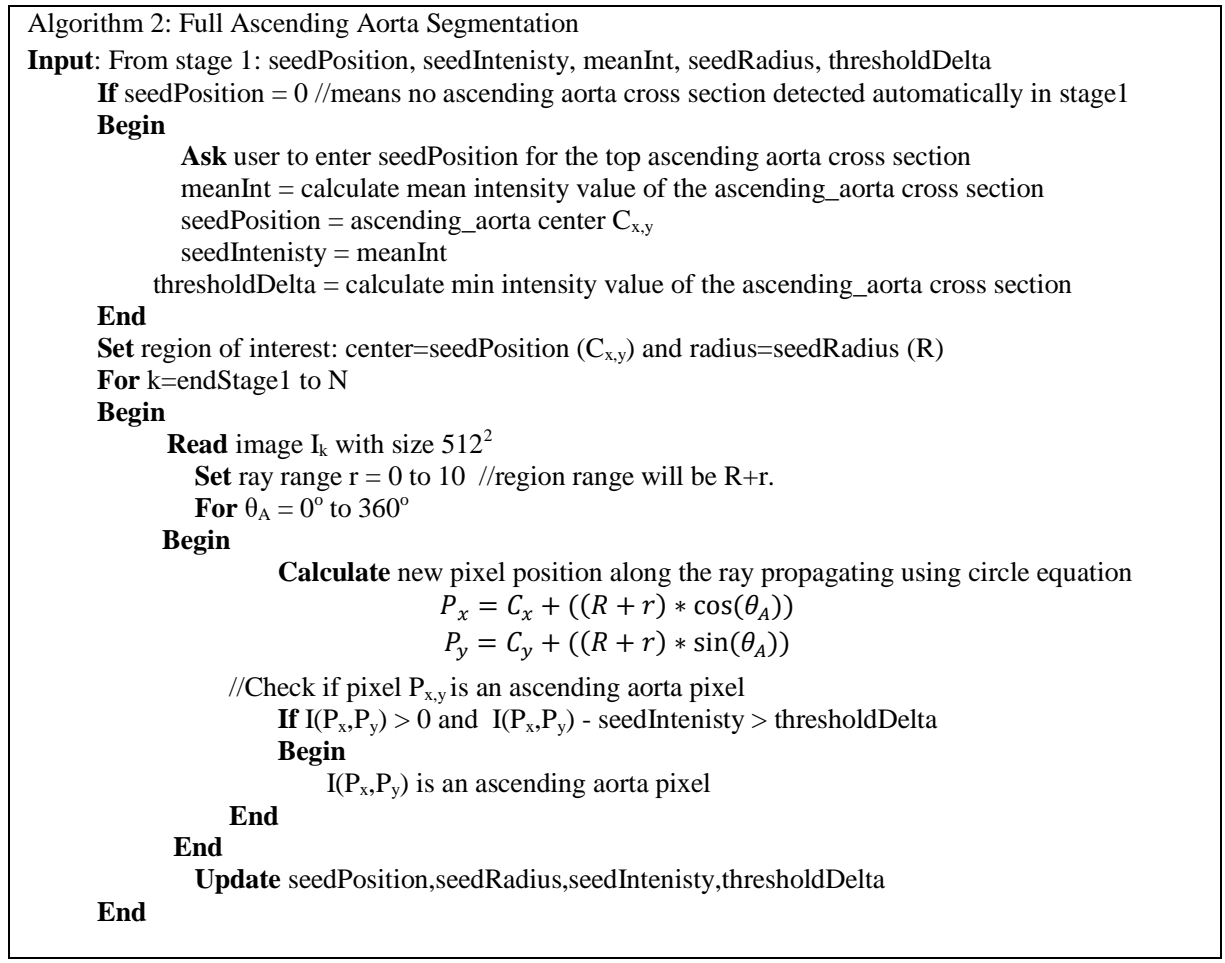

Figure 6. Pseudo Code for Full Ascending Aorta Segmentation Stage 


\section{RESULTS AND DISCUSSION}

\subsection{Datasets}

The proposed automatic ascending aorta segmentation algorithm is applied on two datasets from two imaging modalities: CTA and PC-MRI.

1. Computed Tomography Angiography (CTA): cardiac CTA database provided by the Rotterdam Coronary Artery Algorithm Evaluation Framework [19]. The database includes 48 datasets with a minimum of 253 slices up to a maximum of 640 slices for a volume. The CTA datasets were acquired in the Erasmus MC, University Medical Center Rotterdam, in Netherlands. Some of the datasets were acquired with a 64-slice CT scanner and some with a dual-source CT scanner.

2. Phase Contrast - Magnetic Resonance Imaging (PC-MRI): database of 8 datasets acquired using 1.5 Tesla-MRI scanners [9].

\subsection{Computing Resources}

The implementation is done on an Intel core i5 machine with $2.50 \mathrm{GHz}$ CPU, 6 GB RAM and an NVidia GeForce GT $635 \mathrm{M}$ graphics card. The visualization and rendering for the 3D segmented ascending aorta is done using ImageVis 3D version 3.1.0 [20].

\subsection{Partial Ascending Aorta Segmentation:}

The first stage of the automatic segmentation algorithm begins with Hough transform for Circular Structures Detection. The Hough transform is applied on every CTA/PC-MRI image slice beginning from the cranial third of the input volume. The radius range specified for the Hough transform is set over all tested datasets based on the medical ascending aorta diameter range mentioned in [13]. First when Hough Transform is applied within the specified radius range, both the ascending and descending aorta cross sections are detected see Figure 7(a), 7(d). To identify the ascending aorta from the descending aorta, the proposed ascending aorta model is fitted to the detected circles and the ascending aorta is successfully identified, see Figure 7(b), 7(c). The detected ascending aorta cross section is then segmented and the detection process continues with every consecutive image slice, while the ascending aorta shape is still uniformly circular. The first stage halts when the shape of the ascending aorta deforms. The output of this stage is part of the ascending aorta being segmented; see Figure 7(c).

\subsubsection{CTA Results}

The partial ascending aorta segmentation algorithm as shown in Figure 8 is applied on the 48 CTA datasets and succeeded in all the cases to partially segment the ascending aorta, as shown in table. The number of slices segmented differs per dataset. As observed from table, the number of slices segmented in some datasets is larger than others based on the volume size and the spatial resolution per scanner (since datasets are collected from different scanners).

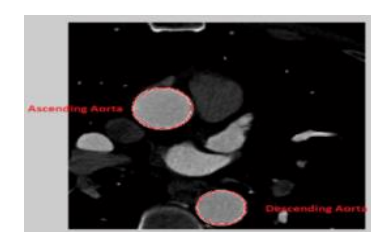

(a)

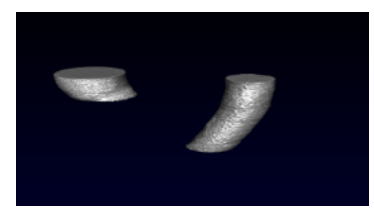

(d)

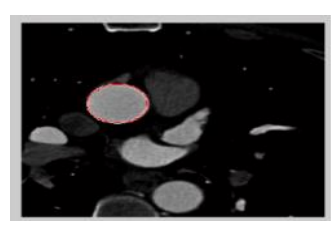

(b)

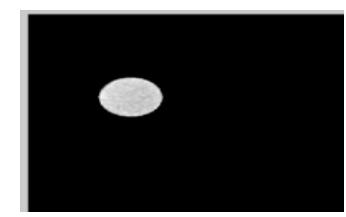

(c)

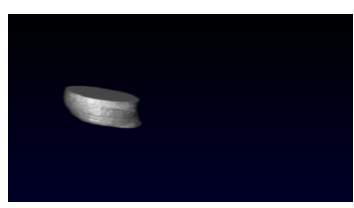

(e)

Figure 7. (a) Ascending Aorta and Descending Aorta are Both Detected on a CTA Image Slice, (b) Ascending Aorta is Identified, (c) Isolated Ascending Aorta, (d) 3D Rendering for the Ascending Aorta and Descending Aorta Detected in a, (e) 3D Rendering for the Ascending Aorta after Augmenting the Ascending Aorta Model with the Hough Transform Algorithm 

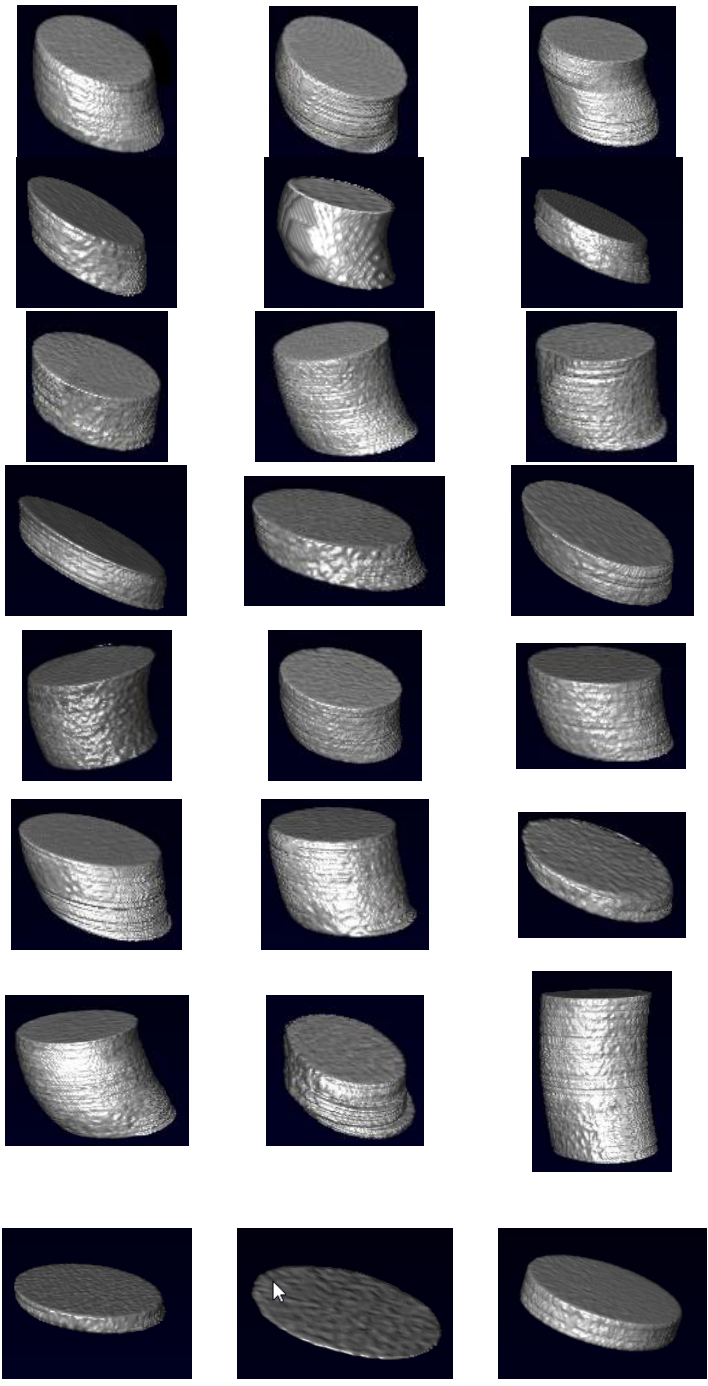
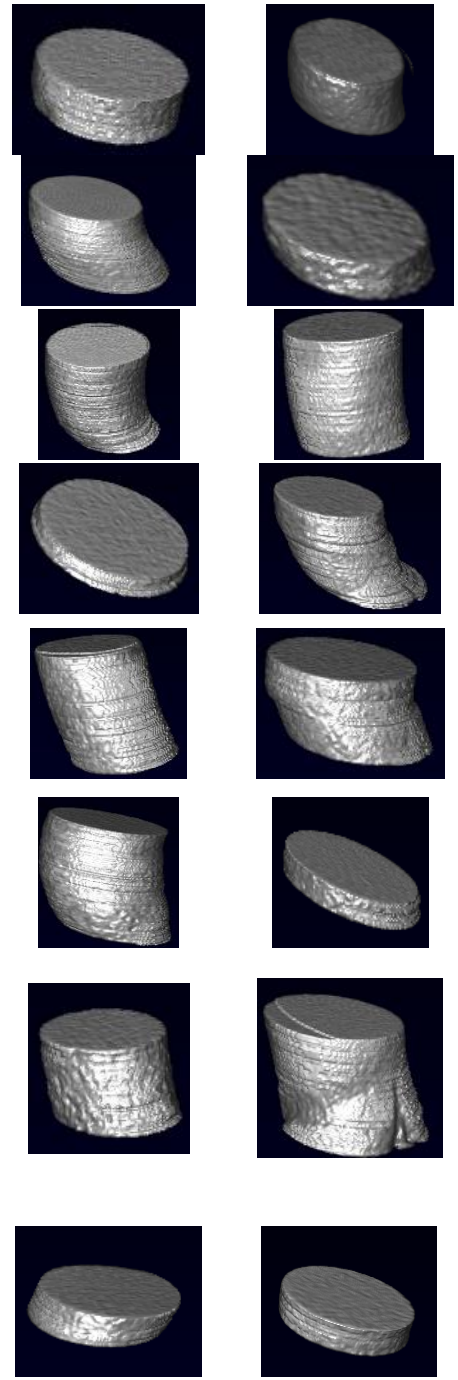

Figure 8. Partial ascending aorta segmentation. The proposed methodology succeeded to identify and detect the ascending aorta in all 48 tested datasets

\subsubsection{PC-MRI Results}

The PC-MRI datasets consist of two image types: magnitude and phase images. Magnitude images determine the anatomical information (presents the spatial resolution), while the phase images encode the velocity $y$ of blood flow in the vessels (presents the temporal resolution) [21]. When applying the partial ascending aorta segmentation algorithm on the PC-MRI data, it succeeded to detect the ascending aorta cross section not only from the magnitude images, but also from phase images as indicated in Figure 9(a)-9(b). Moreover the algorithm succeeded in detecting the ascending aorta in images with artifacts and different resolutions see Figure 9(c)-9(d).

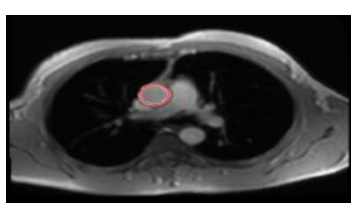

(a)

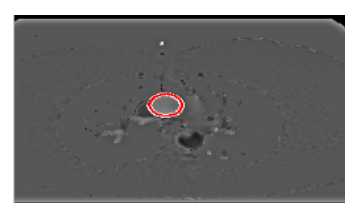

(b)

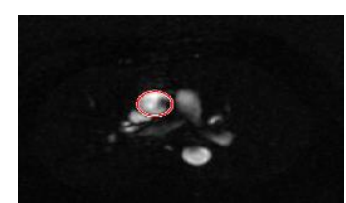

(c)

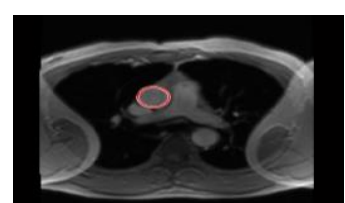

(d)

Figure 9. (a) Ascending Aorta Detected in PC-MRI Magnitude Image, (b) Phase Image, (c) Image with different Resolution, (d) Image with Artifacts 


\subsection{Full Ascending Aorta Segmentation}

When the aorta cross section shape is no more uniformly circular and Hough transform failed to detect it, the proposed ray-search-based propagation region growing algorithm is applied to segment the remaining part of the ascending aorta. The segmented part of the ascending aorta taken from first stage will resemble the seed for the region growing algorithm. The proposed region growing algorithm takes the seed position and intensity to search with from the segmented ascending aorta part.

\subsubsection{CTA Results}

The full ascending aorta algorithm is applied on the remaining unvisited slices of the CTA volumes and it successfully segments the full ascending aorta from the aortic arch down to ostia points as indicated in Figure 10. The threshold used for the region growing is set in all test cases to $I(x, y)>150 \mathrm{HU}$.
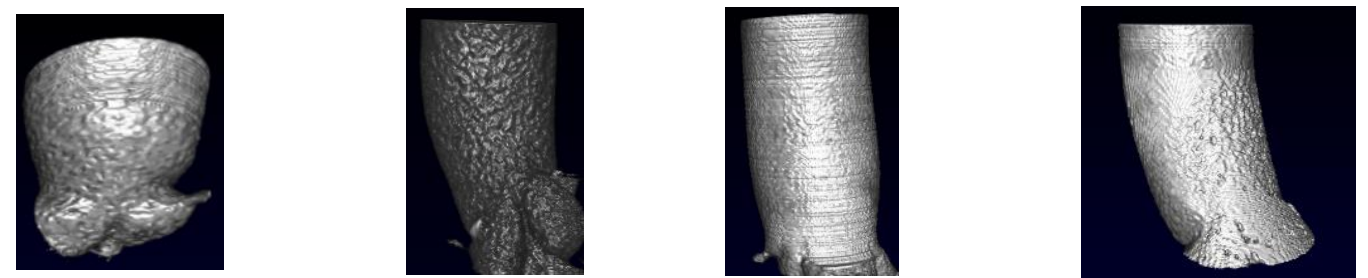

Figure 10. Full Ascending Aorta Segmentation Results for 4 CTA Datasets

\subsubsection{PC-MRI Results}

When applying the full ascending aorta segmentation algorithm on PC-MRI images at different time frames, it succeeded to segment the ascending aorta cross section, although its shape differs from frame to another, see Figure 11(b).
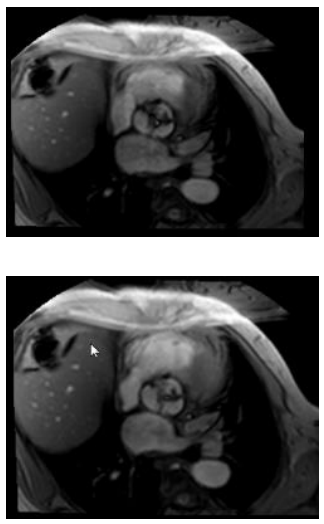

(a)
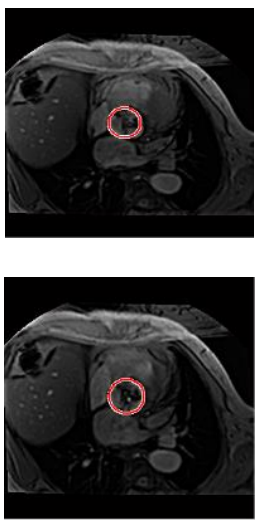

(b)

Figure 11. (a) PC-MRI Images at different Time Frames, (b) Ascending Aorta Detected as Red Circle

\subsection{Performance Measures}

\subsubsection{Accuracy Measure}

To measure the accuracy of the segmentation results, the automatically segmented ascending aorta is validated versus a manually segmented ascending aorta cross sections; using Dice Similarity Coefficient (DSC), to measure the degree of overlap between both cross sections in all the volume slices, see Figure 12.

$$
\text { Dice Coefficient }=\frac{2 * A_{o}}{A_{g}+A_{s}}
$$


where $\mathbf{A}_{\mathbf{g}}$ presents the area of the manually segmented ascending aorta and $\mathbf{A}_{\mathbf{s}}$ presents the area of the automatically segmented ascending aorta and $\mathbf{A}_{\mathbf{o}}$ presents the area of their overlap.

For CTA datasets: dice similarity coefficient is calculated over all test cases with a mean of 0.94 and standard deviation of $0.94 \pm 0.04$, see Table 1 for comparing our accuracy with accuracies mentioned in previous related work.

For PC-MRI datasets: dice similarity coefficient is calculated over all test cases with a mean of 0.97 and standard deviation of $0.94 \pm 0.05$, see Table 1 for comparing our accuracy with accuracies mentioned in previous related work.
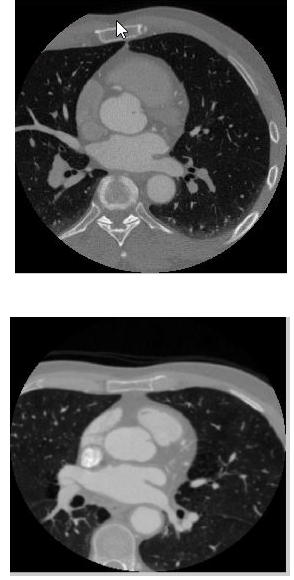

(a)
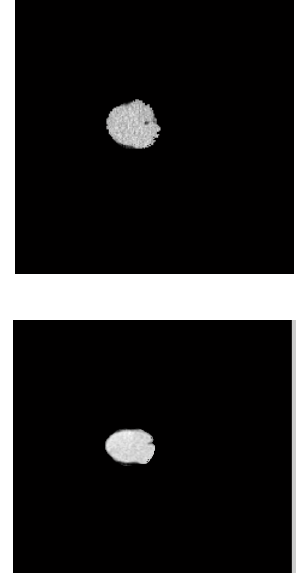

(b)
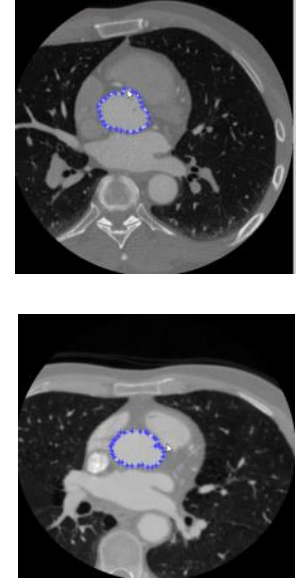

(c)
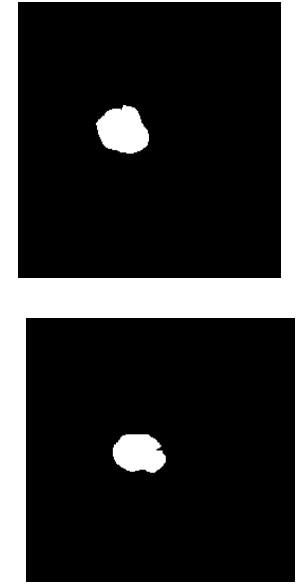

(d)

Figure 12. (a) CTA Image Slices from Two different Datasets, (b) Automatically Segmented Ascending Aorta Cross Sections, (c) Manual Annotation for Ascending Aorta on the CTA Image Slices, (d) Manually Segmented Ascending Aorta Cross Sections

\subsubsection{Processing Time}

The automatic segmentation process takes while processing CTA datasets: a max of 10 seconds for large volumes with size of 560 slices and a min of 7 seconds for volumes with size of 274 slices and for PCMRI datasets it takes 3-4 seconds for volumes of size 14-17 slices. See Table 1, for comparing our processing time with previous related work.

Table 1. Comparing Performance between our Proposed Algorithm and Previous Related Work in Terms of Dataset Used, Automation of the Method, Accuracy of Results and Processing Time

\begin{tabular}{|c|c|c|c|c|}
\hline Algorithm & Dataset & Automation & Accuracy & $\begin{array}{c}\text { Processing Time per } \\
\text { volume processing }\end{array}$ \\
\hline Egger et al. [22] & 50 CTA datasets & semi-automatic & $90.67 \%$ & $30-90$ seconds \\
\hline Xie et al. [2] & $\begin{array}{l}359 \text { low-dose non- } \\
\text { contrast CT images }\end{array}$ & semi-automatic & $93.3 \%$ & ------- \\
\hline Kurugol et al. [23] & $45 \mathrm{CT}$ datasets & automatic & $93.1 \%$ & ------- \\
\hline Al-Agamy et al. [9] & $\begin{array}{c}5 \text { PC-MRI datasets, } \\
\text { containing overall ninety } \\
\text { time frames }\end{array}$ & Semi-automatic & $\begin{array}{c}92.812 \% \text { (for } \\
\text { normal datasets) }\end{array}$ & -- \\
\hline Herment et al. [8] & 52 PC-MRI datasets & automatic & $94.5 \%$ & 3-17 seconds \\
\hline Our Proposed Algorithm & 48 CTA datasets & automatic & $94.72 \%$ & 7-10 seconds \\
\hline & 8 PC-MRI datasets & automatic & $97.13 \%$ & 3-4 seconds \\
\hline
\end{tabular}

From the above table, it is indicated that our proposed algorithm gives better accuracy and processing time with respect to other related work. Our proposed algorithm is fully automatic, applied on two imaging modalities with the same parameters set values and still gives better accuracy and processing time which proves the novelty and reliability of the proposed algorithm. 


\section{CONCLUSION}

In this paper a novel algorithm for automatic segmentation of ascending aorta is presented. The algorithm has been tested on datasets from two imaging modalities: CTA and PC-MRI. First the ascending aorta has been studied anatomically and an ascending aorta model has been built based on its anatomical features. The segmentation algorithm goes through two main stages: Partial and Full ascending aorta segmentation. In the first stage a part of the ascending aorta is segmented using Hough transform for detecting circular/elliptical structures. The ascending aorta is identified from other similar structures through model fitting of the proposed ascending aorta model. The part of the ascending aorta segmented is the part where the ascending aorta has a uniform circular shape in the volume. Once the ascending aorta shape begins to deform, the second stage begins. In the second stage, the remaining part of the ascending aorta is segmented through the proposed center to edge ray-search-based propagation region growing algorithm. The new region growing algorithm gets its seed point and seed intensity value from the first stage. For every new image slice; the search extends in a circular manner as 360 rays, where each ray begins from the seed point given and propagates till hitting the edge of the ascending aorta. The ascending aorta is successfully segmented from both imaging modalities with accuracy of the segmentation having a mean Dice Similarity Coefficient (DSC) of $94.72 \%$ for CTA datasets and $97.13 \%$ for PC-MRI datasets. The processing time for the segmentation took about 7-10 second for CTA datasets and about 3-4 seconds for PC-MRI datasets. The accuracy and time measures have been compared to previous related work and our proposed algorithm proves its competitive performance among other methods. The accuracy attained proves the reliability of the proposed algorithm. Moreover the novelty of the approach lies in its full automation (no user interaction needed) and also no preprocessing steps are needed to identify a region of interest or remove surrounding organs to easily detect the aorta. Moreover, the algorithm works on images from different imaging modalities with the same parameters set values, and this proves its applicability and robustness, and possibility to be extended to other similar problems in the medical image analysis field.

\section{REFERENCES}

[1] M. B. Srichai, et al., "Cardiovascular applications of phase-contrast MRI," AJR. Am. J. Roentgenol., vol/issue: 192(3), pp. 662-75, 2009.

[2] Y. Xie, et al., "Automated aorta segmentation in low-dose chest CT images," Int. J. Comput. Assist. Radiol. Surg., vol/issue: 9(2), pp. 211-219, 2014.

[3] S. Bock, et al., "Robust vessel segmentation," in Medical Imaging, pp. 691539, 2008.

[4] A. Hennemuth, et al., "One-click coronary tree segmentation in CT angiographic images," vol. 1281, pp. 317-321, 2005.

[5] P. H. Kitslaar, et al., "Connected Component and Morpholgy Based Extraction of Arterial Centerlines of the Heart (Cocomo Beach)," pp. 1-8, 2008.

[6] B. Bouraoui, et al., "Fully Automatic 3d Segmentation of Coronary Arteries Based on Mathematical Morphology 2," LINC , UMR ULP-CNRS 7191, pp. 1059-1062, 2008.

[7] S. C. Saur, et al., "Automatic ascending aorta detection in CTA datasets," in Bildverarbeitung für die Medizin 2008, Springer, pp. 323-327, 2008.

[8] A. Herment, et al., "Automated segmentation of the aorta from phase contrast MR images: Validation against expert tracing in healthy volunteers and in patients with a dilated aorta," J. Magn. Reson. Imaging, vol/issue: 31(4), pp. 881-888, 2010.

[9] A. O. Al-Agamy, et al., "Segmentation of ascending and descending aorta from magnetic resonance flow images," 2010 5th Cairo Int. Biomed. Eng. Conf. CIBEC 2010, vol. 1, pp. 41-44, 2010.

[10] “Ascending Aorta,” [Online]. Available: https://en.wikipedia.org/wiki/Ascending_aorta. [Accessed: 05-Sep-2016].

[11] C. Wang and O. Smedby, "An automatic seeding method for coronary artery segmentation and skeletonization in CTA," Insight J., pp. 1-8, 2008.

[12] Y. Zheng, et al., "Automatic Aorta Segmentation and Valve Landmark Detection in C-Arm CT for Transcatheter Aortic Valve Implantation," vol/issue: 31(12), pp. 2307-2321, 2012.

[13] R. Erbel and H. Eggebrecht, "Aortic dimensions and the risk of dissection," Heart, vol/issue: 92(1), pp. 137-142, 2006.

[14] "Hounsfield scale," [Online]. Available: https://en.wikipedia.org/wiki/Hounsfield_scale. [Accessed: 05-Sep-2016].

[15] S. C. Saur, et al., "Automatic Ascending Aorta Detection in CTA Datasets."

[16] P. H. Kitslaar, et al., "Connected Component and Morpholgy Based Extraction of Arterial Centerlines of the Heart (Cocomo Beach)," in The MIDAS Journal: MICCAI Workshop--Grand Challenge Coronary Artery Tracking, 2008.

[17] R. K. K. Yip, et al., "Modification of Hough transform for circles and ellipses detection using a 2-dimensional array," Pattern Recognit., vol/issue: 25(9), pp. 1007-1022, 1992.

[18] R. Adams and L. Bischof, "Seeded region growing," Pattern Anal. Mach. Intell. IEEE Trans., vol/issue: 16(6), pp. 641-647, 1994.

[19] M. Schaap, et al., "Standardized evaluation methodology and reference database for evaluating coronary artery centerline extraction algorithms," vol. 13, pp. 701-714, 2009. 
[20] CIBC, "ImageVis3D: An interactive visualization software system for large-scale volume data.," 2014. [Online]. Available: http://www.imagevis3d.org.

[21] M. B. Srichai, et al., "Cardiovascular applications of phase-contrast MRI," Am. J. Roentgenol., vol/issue: 192(3), pp. 662-675, 2009.

[22] J. Egger, et al., "Aorta Segmentation for Stent Simulation,” pp. 10, 2011.

[23] S. Kurugol, et al., "Aorta Segmentation with a 3D Level Set Approach and Quantification of Aortic Calcifications in Non-Contrast Chest CT," pp. 2343-2346, 2012. 